\title{
Molecular Cloning and Expression Profile of Class $E$ Genes Related to Sepal Development in Nelumbo nucifera
}

\author{
Zhuoxing Liu ${ }^{1,2, \dagger}$, Dasheng Zhang ${ }^{1,+}{ }^{+}$Weiwei Zhang ${ }^{3}$, Lei Xiong ${ }^{1,2}$, Qingqing Liu ${ }^{1}$, Fengluan Liu ${ }^{2}$, \\ Hanchun Li ${ }^{1}$, Xiangjie An ${ }^{1}$, Lijie Cui ${ }^{2, *}$ and Daike Tian ${ }^{1, *}$ \\ 1 Shanghai Key Laboratory of Plant Functional Genomics and Resources, Shanghai Chenshan Plant Science \\ Research Center of Chinese Academy of Sciences, Shanghai Chenshan Botanical Garden, \\ Shanghai 201602, China; 1000459190@smail.shnu.edu.cn (Z.L.); dszhang@cemps.ac.cn (D.Z.); \\ 1000479715@smail.shnu.edu.cn (L.X.); nicecocoliu@sina.com (Q.L.); lihanchun1022@126.com (H.L.); \\ anxiangjie@cemps.ac.cn (X.A.) \\ 2 Development Center of Plant Germplam Resources, College of Life Science, Shanghai Normal University, \\ Shanghai 200234, China; 19liu19@163.com \\ 3 Department of Plant Science and Technology, Shanghai Vocational College of Agriculture and Forestry, \\ Shanghai 201699, China; zhangww@shafc.edu.cn \\ * Correspondence: cuilj@shnu.edu.cn (L.C.); dktian@cemps.ac.cn (D.T.); Tel.: +86-21-37792288-932; \\ Fax: +86-21-57762652 \\ + These authors contributed equally to this work.
}

check for updates

Citation: Liu, Z.; Zhang, D.; Zhang, W.; Xiong, L.; Liu, Q.; Liu, F.; Li, H.; An, X.; Cui, L.; Tian, D. Molecular Cloning and Expression Profile of Class E Genes Related to Sepal Development in Nelumbo nucifera. Plants 2021, 10, 1629. https://doi.org/10.3390/ plants10081629

Academic Editor: Baohong Zhang

Received: 2 July 2021

Accepted: 21 July 2021

Published: 9 August 2021

Publisher's Note: MDPI stays neutral with regard to jurisdictional claims in published maps and institutional affiliations.

Copyright: (c) 2021 by the authors. Licensee MDPI, Basel, Switzerland. This article is an open access article distributed under the terms and conditions of the Creative Commons Attribution (CC BY) license (https:/ / creativecommons.org/licenses/by/ $4.0 /)$.
Abstract: The lotus (Nelumbo Adans.) is an important aquatic plant with ornamental, medicinal and edible values and cultural connotations. It has single-, semi-double-, double- and thousandpetalled types of flower shape and is an ideal material for developmental research of flower doubling. The lotus is a basal eudicot species without a morphological difference between the sepals and petals and occupies a critical phylogenetic position in flowering plants. In order to investigate the genetic relationship between the sepals and petals in the lotus, the class $E$ genes which affect sepal formation were focused on and analyzed. Here, SEPALLATA 1(NnSEP1) and its homologous genes AGAMOUS-LIKE MADS-BOXAGL9 (NnAGL9) and MADS-BOX TRANSCRIPTION FACTOR 6-like (NnMADS6-like) of the class $E$ gene family were isolated from the flower buds of the Asian lotus (Nelumbo nucifera Gaertn.). The protein structure, subcellular localization and expression patterns of these three genes were investigated. All three genes were verified to locate in the nucleus and had typical MADS-box characteristics. NnSEP1 and NnMADS6-like were specifically expressed in the sepals, while NnAGL9 was highly expressed in the petals, suggesting that different developmental mechanisms exist in the formation of the sepals and petals in the lotus. The significant functional differences between NnSEP1, NnMADS6-like and NnAGL9 were also confirmed by a yeast twohybrid assay. These results expand our knowledge on the class $E$ gene family in sepal formation and will benefit fundamental research on the development of floral organs in Nelumbo.

Keywords: MADS-box; SEPALLATA; AGL9; MADS6-like; sepal; petal; Nelumbo

\section{Introduction}

Plant floral identity organs are controlled by a series of homeomorphic genes and almost allomorphic genes related to flower organ development that belong to the family of MADS-box genes [1]. The MADS-box gene family has become an essential part of the formation of plant floral organs. The MADS-box gene family is a crucial transcription factor with a highly conserved MADS domain that widely presents in various organisms [2-4]. The MADS-box gene family can be divided into two groups: type I and type II [5]. The type II genes comprise the MIKC-type genes of plants and the MEF2-like genes of animals and fungi. Moreover, the MIKC-type proteins own an extremely conserved MADS domain and three moderately conserved domains (I, K and C) [6]. The various members of the MADS-box gene family have been known to participate predominantly in developmental 
processes, such as plant vegetative reproduction [7,8], flower organ formation [9-11], male and female gametophyte development [12] and fruit development [13].

$A n A B C$ model of flower development was proposed by a previous report [14]. The $A B C$ molecular regulation model indicates that the four whorl organs from the outside to the inside are controlled by three types of gene. For example, the $A$ gene controls the sepals alone, $A+B$ control the petals, $B+C$ control the stamens and $C$ controls the pistils alone. Simultaneously, the $A$ and $C$ genes repress each other [14]. Then, the ABCDE model and the quartet model of flower development were proposed and widely recognized by researchers [15-17]. The ABCDE model proposes that floral organs can be subdivided into five different classes of sepals, petals, stamens, pistils and ovules. The formation of flower organs depends on a protein tetramer encoded by five types of genes. The quartet model could accurately describe the interaction of five types of transcription factors. It also could explain the mechanism of flower phenotypes because of the loss of transcription factor genes [17].

Class $E$ genes are central to the sepals and to regulating the formation of floral organs in each round. The SEPALLATA (SEP) gene belongs to class $E$ and is a separate subfamily of the MADS-box gene family $[18,19]$. Previous studies showed that the triple mutants SEP1/2/3 in Arabidopsis caused all flower organs to turn into sepals. Additionally, the $S E P$ gene could shorten the vegetative growth period and promote the early flowering of plants $[11,18,20-22]$. Besides the genes in the ABCDE model, other MADS-box genes are also responsible for sepal development. The AGL6 gene subfamily in plants is ancient and widespread [23]. Phylogenetic analysis shows that the AGL6 homologous gene belongs to the super branch of AP1/AGL9 (including AGL6, SEP and SQUA) [24]. The two branches of AGL6 and SEP represent sister relationships to each other, and the domains at the Cterminal are extremely similar [25]. It has been speculated that the AGL6 homologous gene and the SEP gene are similar in function [26].

The lotus (Nelumbo Adans.) is a perennial aquatic plant and one of the top ten most famous flowers in China. It is an important crop with ornamental, medicinal and edible uses and cultural connotations. In addition, it is one of the earlier plants in the origin of angiosperms and is considered as a 'living fossil'. The lotus is divided into two species according to its geographical location and morphological differences: the American lotus (N. lutea Willd.), and the Asian lotus (N. nucifera Gaertn.). The American lotus has a single flower only, while the Asian lotus has a variety of floral shapes, including single-, semidouble-, double- and thousand-petalled types (Figure 1). No reproductive isolation occurs between the two species and among most cultivars of the lotus; therefore, it is a good material for studying floral development. In Nelumbo, the phenotype of the outermost sepals (usually four) is similar to the inner petals, meaning breeders usually use the concept of 'tepal' instead of 'sepal' for petals in the lotus. In order to investigate the genetic relationship between sepals and petals in the lotus, the class $E$ genes, which affect sepal formation, were focused on and analyzed. In this study, a single-petalled type of wild Asian lotus was used as the experimental material. Using the RT-PCR method, the SEP gene and its homologous genes relating to sepal formation were cloned, and a series of bioinformatics and function analyses was performed. 

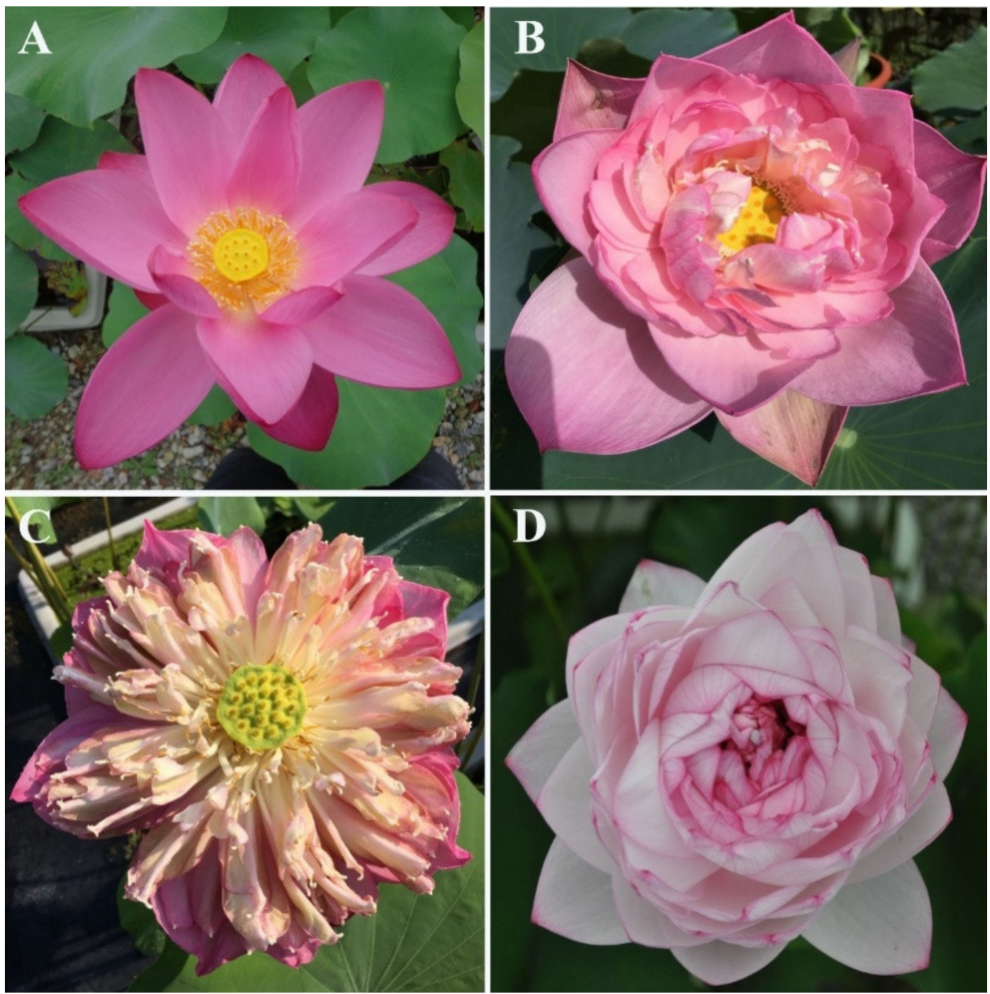

Figure 1. The floral forms of Nelumbo nucifera. (A) Single form; (B) semi-double form; (C) double form; (D) thousand-petalled form.

\section{Materials and Methods}

\subsection{Experimental Materials}

The wild type of the Asian lotus (Figure S1) was used as the experimental material. It was collected from Weishan Lake in Shandong Province, China, and cultivated in the International Nelumbo Collection, which is located at Shanghai Chenshan Botanical Garden. The root, pedicel, leaf, petal, stamen, pistil, receptacle and rhizome of the Asian lotus at the flower maturation stage were sampled for analysis of genes' expression patterns. Following sample collection, all the samples were immediately treated by liquid nitrogen freezing and stored at $-80^{\circ} \mathrm{C}$ for subsequent uses.

\subsection{Total RNA Extraction and $c D N A$ Synthesis}

The RNA of the root, pedicel, leaf, petal, stamen, pistil, receptacle, rhizome and flower was extracted according to the instructions of the RNAprep Pure Plant Kit (TIANGEN, DP441, Beijing, China). The quality and purity of RNA samples were determined using the Thermo ND2000c (Thermo Fisher Scientific, American). The reverse transcription reaction was performed according to the instructions of the High Efficiency Reverse Transcription Kit (TOYOBO, FSK-100, Osaka, Japan).

\subsection{Gene Clone and Sequence Identification}

From the NCBI (https:/ / www.ncbi.nlm.nih.gov /, accessed on 9 January 2021), only one SEP gene (XM_010259656) was predicted to belong to the class $E$ gene family. The total RNA of the Asian lotus flower bud $(<2 \mathrm{~cm})$ was used as a template for high-fidelity amplification. After the reverse transcription with the primer AP, NnSEP1-full-F and AUAP were designed to amplify the full length of the SEP gene with KOD-Plus public high-fidelity kit (KOD-201, Japan) (the primers are listed in Table 1). The PCR products were sequenced by Qingke Biotechnology Company (Shanghai, China). 
Table 1. The primers used in this study.

\begin{tabular}{cc}
\hline Primer Name & Sequence (5' $\rightarrow \mathbf{3}^{\prime} \mathbf{)}$ \\
AP & GGCCACGCGTCGACTAGTACTTTTTTTTTTTTTTTT \\
NnSEP1-full-F & GTACTAGTCGACGCGTGGCC \\
NnSEP1-RT-F & ATGGGGAGAGGAAGGGTAGA \\
NnSEP1-RT-R & GGAAGCTGGATGAAAGTAGT \\
NnAGL9-RT-F & AAGCATCCACCCAGGAATAT \\
NnAGL9-RT-R & AGGGAGTCAGAGGAGCTGAG \\
NnMADS6-like-RT-F & CAATGCCCTGTTAGCTTCGC \\
NnMADS6-like-RT-R & AGCTAGGCAACGGAAGACAC \\
NnActin-F & TCCCTCCGGTGGAACATAGT \\
NnActin-R & ACCACTGCTGAACGGGAAAT \\
1300-NnSEP1-GFP-F & ATGGCTGGAATAGAACCTCA \\
1300-NnSEP1-GFP-R & GGATCCATGGGGAGAGGAAGGGTAGA \\
1300-Nn AGL9-GFP-F & TCTAGAAAGCATCCACCCAGGAA \\
1300-Nn AGL9-GFP-R & GGATCCATGGGGAGAGGTAGGGTTGA \\
1300-NnMADS6-like-GFP-F & TCTAGATGCCAGCCACCCAGGC \\
AD-NnSEP1-F & GGATCCATGGGAAGAGGACGAGTAGA \\
AD-NnSEP1-R & TCTAGAGAGAACCCATCCTTGAAT \\
AD-NnAGL9-F & CATATGGGGAGAGGAAGGGTAGAGCTGAA \\
AD-NnAGL9-R & GGATCCCAAGCATCCACCCAGGAATATAACCATT \\
AD-NnMADS6-like-F & CATATGGGGAGAGGAAGGGTAGAGCTGAA \\
AD-NnMADS6-like-R & GGATCCCTGCCAGCCACCCAGGCATATAACTA \\
& CATATGGGGAGAGGAAGGGTAGAGCTG \\
\hline & GGATCCCGAGAACCCATCCTTGAATGAAGTTA \\
\hline
\end{tabular}

\subsection{Sequence Analysis}

Homologous sequence analysis was performed online using Blast provided by NCBI, and the open reading frames of cloned genes were searched online using ORF Finder. Sequence analysis and amino acid translation were performed using DNAMAN 6.0 software. InterPro (http:/ /www.ebi.ac.uk/interpro/search/sequence, accessed on 1 February 2021) was used to predict the protein domain. PtotParam provided by ExPASY (https: / / expasy.org/ proteomics, accessed on 5 February 2021) was used to analyze the physicochemical properties of the protein online. ProtScale was used to analyze the hydrophobicity of the protein. NetPhos 2.0 Server (http:/ / www.cbs.dtu.dk/services/NetPhos-2.0/, accessed on 6 February 2021) was used to predict the phosphorylation site. GOR4 was used to predict the secondary structure of the protein. SWISS-MODEL was used to predict the tertiary structure of the protein. PSORT (https:/ / www.genscript.com/tools/psort, accessed on 18 February 2021) provided by GenScript was used to perform subcellular localization prediction online. TMHMM (http://www.cbs.dtu.dk/services/TMHMM/, accessed on 5 February 2021) was used to perform transmembrane domain analysis. DNAMAN 6.0 software was used for multiple sequence alignment, and MAGE 5.0 software was used to construct the phylogenetic tree.

\subsection{Subcellular Localization}

The full length of NnSEP1, NnAGL9 and NnMADS6-like genes without an amino acid stop code was amplified by PCR (primers are listed in Table S1). Then, the fragments were digested with BamH I, and Xba I was ligated with the pCAMBIA 1300-GFP vector with the same digestion to construct the fusion vectors pCAMBIA1300-NnSEP1-GFP, pCAMBIA1300-NnAGL9-GFP and pCAMBIA1300-NnMADS6L-GFP. The fusion plasmids were transformed into Agrobacterium GV3101 competent cells, and the positive clones were selected for transformation into Nicotiana benthamiana for the transient expression test. Nicotiana benthamiana was grown in a greenhouse under the conditions of $25^{\circ} \mathrm{C}, 70 \%$ relative humidity and long day ( $16 \mathrm{~h}$ light and $8 \mathrm{~h}$ dark) for subcellular localization experiments. The pellets were resuspended in $200 \mathrm{~mL}$ MS containing $200 \mu \mathrm{M}$ acetosyringone and $0.39 \mathrm{~g}$ 
MES at $28{ }^{\circ} \mathrm{C}$ and were incubated in a shaker at $140 \mathrm{rpm}$ for $2-3 \mathrm{~h}$ until $\mathrm{OD}_{600}$ reached 0.6. Two Agrobacterium GV3101 strains carrying the recombinant expression plasmid and control were injected into tobacco leaves. The treated $N$. benthamiana was cultured in the dark for $24 \mathrm{~h}$ and then in the light for $24 \mathrm{~h}$ at $25^{\circ} \mathrm{C}$. The dorsal epidermis of the leaf blade was collected, and the GFP fluorescence signal was detected by a laser confocal microscope (Olympus, FV10i), as described in a previous report [27].

\subsection{Gene Expression Analysis}

The gene expression in the root, stem, leaf, petal, stamen, pistil, receptacle, rhizome and flower buds was detected using the real-time quantitative RT-PCR method, using NnACTIN as the internal reference gene [28]. The RT-PCR primers are listed in Table 1. The quantitative reactions were performed based on the SYB Green design manual (TAKARA, DRR820A). The quantitative reaction was performed on an ABI Step ONE quantitative PCR instrument, using the $\left(2^{-\Delta \Delta C T}\right)$ method to calculate the relative expression level. Each experiment was replicated three times ( 3 biological and 3 technical repeats).

\subsection{Yeast Two-Hybrid (Y2H) Assay}

A yeast two-hybrid assay of the GAL4 system was used, as described in a previous report [29]. The full-length coding sequences of NnSEP1, NnAGL9 and MADS6-like were cloned and constructed into the pGBKT7 and pGADT7 vectors (the primers are listed in Table 1). The bait and prey fusion plasmid were co-transformed into strain AH109 using the lithium acetate method. The isolated colonies were incubated on SD selection medium (SD/ - Trp / - Leu and SD/-Trp/-Leu/-His/-Ade/+X- $\alpha$-gal) plates in triplicates for interaction analysis.

\section{Results}

\subsection{Cloning of the Full-Length cDNA of the SEP Gene}

Only SEP1 was predicted to belong to the class $E$ gene family in the lotus genome database (https: / / www.ncbi.nlm.nih.gov / nuccore /XM_010259656.2, accessed on 9 January 2021). According to the sequence predicted by the database, the specific primers NnSEP1-full-F and AUAP were designed. After the reverse transcription with the primer AP, the designed NnSEP1-full-F and AUAP were used to amplify the full length of the SEP1 gene (the primers are list in Table 1).

A single band of about $900 \mathrm{bp}$ was obtained by the PCR method (Figure 2A), which was close to the size predicted in the lotus genome. After the band was recovered and cloned into the blunt vector, we sequenced it and found that this band was a mixture of several gene fragments, the SEP1 gene and two new genes (NnAGL9 and NnMADS6-like) with high homology to the SEP1 gene.

The open reading frame (ORF) of the NnSEP1 gene was $732 \mathrm{bp}$ in length and encoded 243 amino acids. The BLAST analysis of the homologous sequence showed that the gene had homology with the SEP1 gene of other plants. The ORF of the NnAGL9 gene was $720 \mathrm{bp}$ in length and encoded 239 amino acids. The BLAST analysis of the homologous sequence showed that the gene has homology with the SEP3 gene in other plants. The ORF of the NnMADS6-like gene was $729 \mathrm{bp}$ in length and encoded 242 amino acids. The BLAST analysis of the homologous sequence revealed that the NnMADS6-like gene had homology with other plant $A G L 6$ genes.

DNAMAN software was used to compare the amino acid sequences of NnSEP1, NnAGL9 and NnMADS6-like (Figure 2E). All three proteins had the characteristics typical of MADS-box genes. The MADS-box domain at the $5^{\prime}$ end was highly conserved, and the middle K-box domain was conserved, while the $3^{\prime} \mathrm{C}$-terminus was quite different. 

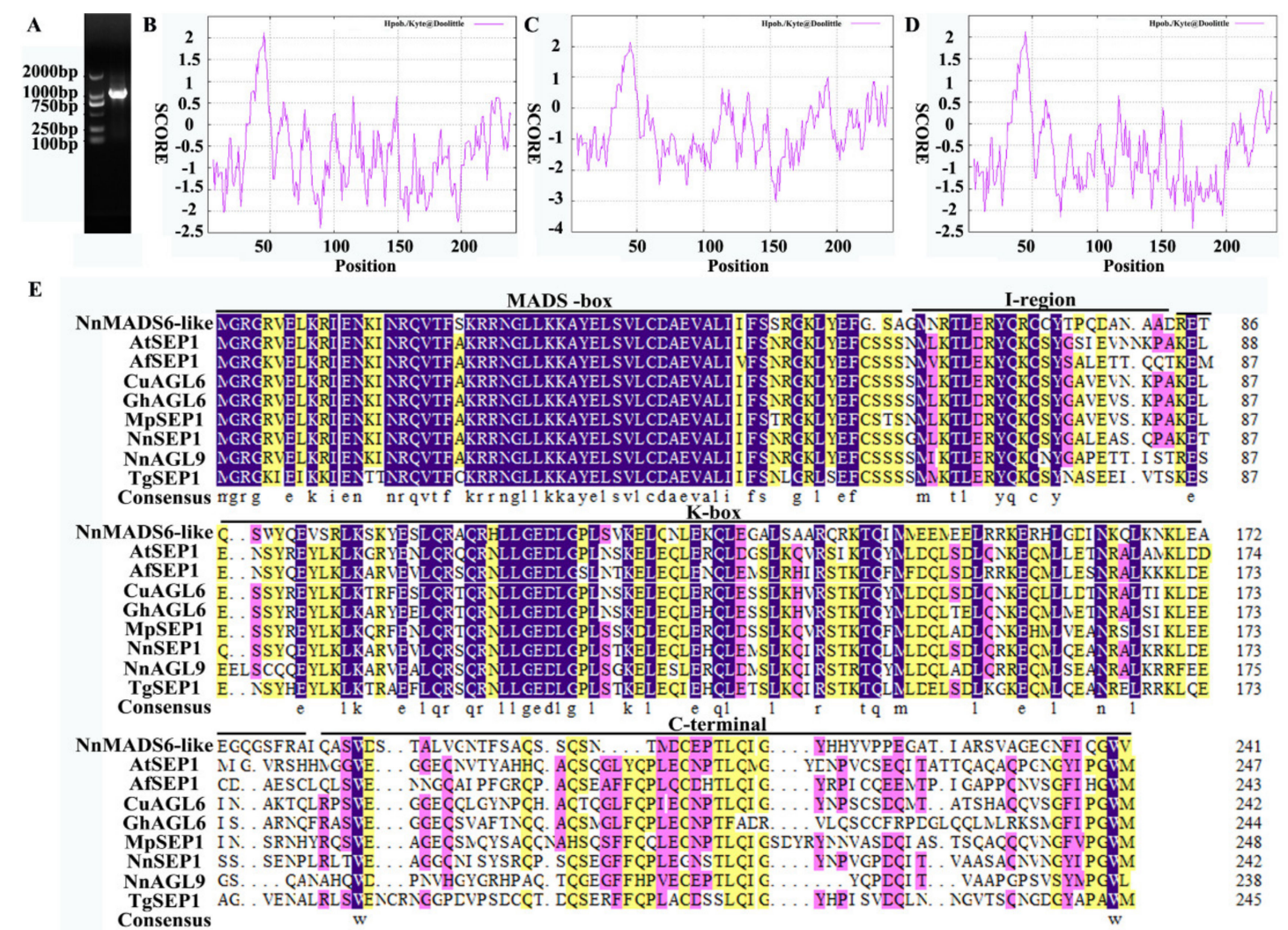

Figure 2. Cloning of three NnSEP genes and predictive analysis of their protein domains. (A) Cloning of the full-length cDNA of three NnSEP genes. (B) The prediction analysis of ProtScale protein hydrophobicity of NnSEP1. (C) The prediction analysis of ProtScale protein hydrophobicity of NnMADS6-like. (D) The prediction analysis of ProtScale protein hydrophobicity of NnAGL9. (E) Sequence alignment of SEP protein in Nelumbo nucifera and other species. The protein sequences of NnSEP1, NnMADS6-like and NnAGL9 aligned were retrieved from NCBI. Different species and matching GenBank accession numbers are shown below. AtSEP1 from Arabidopsis thaliana (AAU82007.1), AfSEP1 from Aristolochia fimbriata (ALV83431.1), CuAGL6 from Citrus unshiu (GAY42048.1), GhAGL6 from Gossypium hirsutum (XP_040972459.1), MpSEP1 from Medicago polyceratia (AFU81295.1), TgSEP1 from Tulipa gesneriana (AQR58150.1).

\subsection{Analysis of Physicochemical Properties of Proteins}

The molecular weights of the NnSEP1, NnAGL9 and NnMADS6-like proteins were all about $27 \mathrm{kDa}$, and their theoretical isoelectric points were 9.15, 8.76 and 9.12, respectively. The total average values of the three proteins' hydrophilicity by ProtParam analysis were $-0.653,-0.703$ and -0.696 , respectively. This indicates that they all remained with hydrophilic protein characters. The result of ProtScale software analysis was the same as that of ProtParam analysis. The prediction analysis of ProtScale protein hydrophobicity was divided according to the score (Figure 2B-D). The NnSEP1, NnAGL9 and NnMADS6-like proteins all remained with hydrophilic proteins. The maximum score of the three proteins was 2.122 , locating at the 45 th amino acid. The minimum values were $-2.389,-2.422$ and -3.033, locating at amino acids 89, 174 and 154, respectively. NetPhos 2.0 Server showed that there were 11 serine phosphorylation sites for NnSEP1, 1 threonine and 7 serine phosphorylation sites for NnAGL9 and 1 tyrosine and 9 serine phosphorylation sites for NnMADS6-like.

\subsection{Secondary and Tertiary Structures of Proteins}

Analysis by GOR4 software showed that the NnSEP1, NnAGL9 and NnMADS6-like proteins were composed of three typical secondary structures of $\alpha$-helix, random coils and extended chains. Simultaneously, the $\alpha$-helix structure accounts for more than $50 \%$. The SWISS-MODEL platform was used to predict the tertiary structure of the three proteins, 
NnSEP1, NnAGL9 and NnMADS-like. The three proteins were mainly composed of an $\alpha$-helix structure, which was consistent with the secondary structure prediction results (Figure 3). The structures of the NnSEP1, NnAGL9 and NnAGL6 proteins in the lotus were very similar.
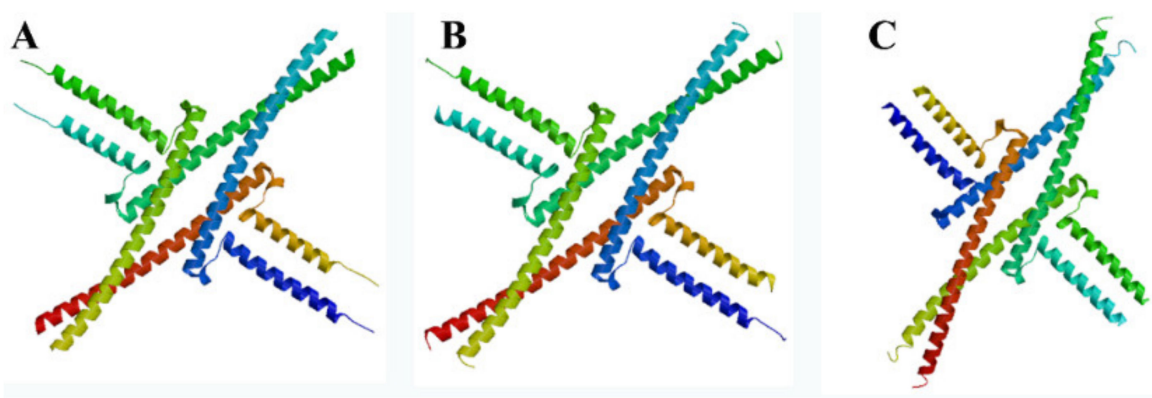

Figure 3. The 3D structural model of proteins. (A) NnSEP1; (B) NnAGL9; (C) NnMADS6-like.

\subsection{Phylogenetic Analysis of NnSEP1, NnAGL9 and NnMADS6-Like Proteins}

In order to determine the classification of the NnSEP1, NnAGL9 and NnMADS6like proteins, the amino acid sequences of NnSEP1, NnMADS6-like and NnAGL9 were used as a template to query the protein database from the NCBI (http:/ / www.ncbi.nlm. nih.gov/blast/Blast.cgi, accessed on 9 January 2021). Through sequence comparison, 32 protein sequences were selected with more than $68 \%$ identity to NnSEP1, NnMADS6like and NnAGL9. Clustal W was used for aligning protein sequences. The phylogenetic relationship was finally analyzed using MEGA 5.0 software [30-32].

The constructed phylogenetic trees were divided into two clans: NnMADS6-like and NnSEP1/NnAGL9 (Figure 4). NnSEP1's closest genetic relationship is with SEP1 of Platanus $\times$ hispanica and Euptelea pleiosperma. NnAGL9 belongs to the SEP3 class, which was divided into two branches. One branch was monocotyledonous plants containing Lolium perenne, Oryza sativa, Zea mays and Hordeum vulgare. The other was dicotyledon. NnAGL9 was first clustered together with the SEP3 protein of Euptelea pleiosperma and then took part in a big branch with other dicotyledon SEP3 classes. Similarly, NnMADS6-like was also the first to gather together with the AGL6 protein of Euptelea pleiosperma and Bocconia frutescens and then joined a large branch with other dicotyledons.

The phylogenetic tree was constructed using the neighbor joining method. Boot strap percentages are shown at dendrogram branch points. The different species and matching GenBank accession numbers are as follows: TcAGL9 from Theobroma cacao (XP_007043947.1), DzAGL9 from Durio zibethinus (XP_022724906.1), MeAGL9 from Manihot esculenta (XP_021631286.1), RcAGL9 from Ricinus communis (XP_015572264.1), CaSEP3 from Coffea arabica (AHW58034.1), PtSEP3 from Pachysandra terminalis (ADC79703.1), EaAGL9 from Eschscholzia californica (AAX15918.1), EpSEP3 from Euptelea pleiosperma (ADC79706.1), ZmMADS6 from Zea mays (NP_001105153.1), OsMADS7 from Oryza sativa (P0C5B0.2), LpMADS5 from Lolium perenne (AAO45877.1), HvAGL9 from Hordeum vulgare (AAS48129.1), MpSEP1 from Medicago polyceratia (AFU81295.1), PeSEP1 from Passiflora edulis (AET98846.1), AtSEP1 from Arabidopsis thaliana (AAU82007.1), GhSEP1 from Gossypium hirsutum (AEL33631.1), MeSEP1 from Manihot esculenta (XP_021592871.1), AfSEP1 from Aristolochia fimbriata (ALV83431.1), AtSEP1 from Akebia trifoliata (ADC79694.1), EpSEP1 from Euptelea pleiosperma (ADC79707.1), PhSEP1 from Platanus $\times$ hispanica (ADR83588.1), LpMADS4 from Lolium perenne (AAO45876.1), HvAGL6 from Hordeum vulgare (AAS48128.1), OsMADS6 from Oryza sativa (Q6EU39.1), AtAGL6 from Arabidopsis thaliana (NP_182089.1), MpAGL6 from Magnolia patungensis (ATB53135.1), CpAGL6 from Chimonanthus praecox (ACN88212.1), MwAGL6 from Magnolia wufengensis (AOZ15901.1), EsAGL6 from Epimedium sagittatum (AEX58638.1), NdAGL6 from Nigella damascena (ALM95509.1), EpSEP1 from Euptelea pleiosperma (ADC79707.1), BfAGL6 from Bocconia frutescens (AOC50668.1). 


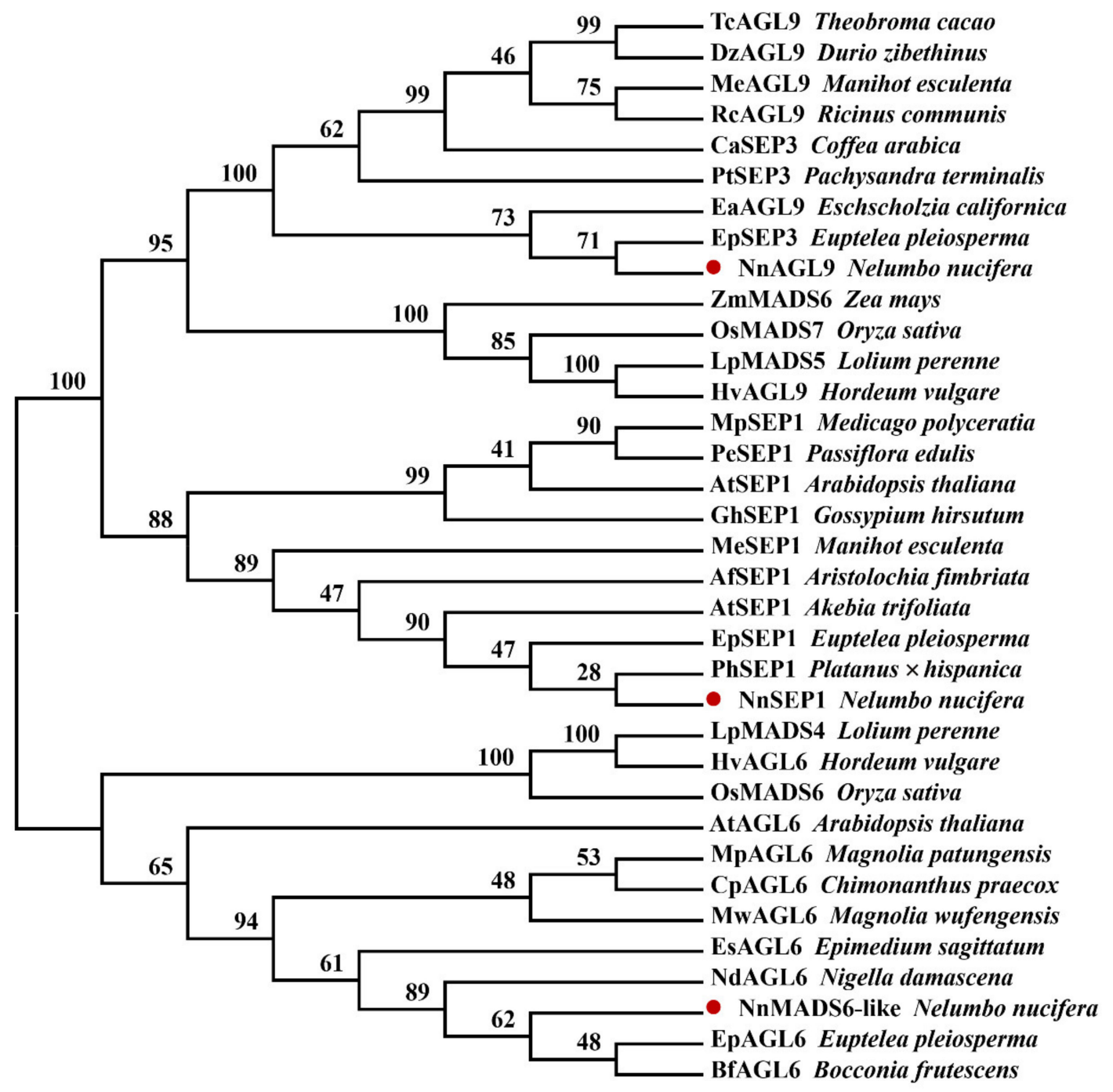

Figure 4. Phylogenetic analysis of NnSEP1, NnAGL9 and NnMADS6-like and their homologous sequences from various plant species.

\subsection{Subcellular Localization of NnSEP1, NnAGL9 and NnMADS6-Like Proteins}

Prediction analysis of the subcellular localization with PSORT showed that NnSEP1, NnAGL9 and NnMADS6-like were mainly distributed in the nucleus. The TMHMM transmembrane domain speculated that neither NnSEP1, NnMADS6-like nor NnAGL9 had a transmembrane signal peptide. Such localization patterns are consistent with the existence of the MADS-box gene family as the transcription factors. To further confirm this, NnSEP1, NnMADS6-like and NnAGL9 were fused with the green fluorescent protein (GFP) and transformed into tobacco leaves for localization analysis. After observation under a laser confocal microscope, NnSEP1, NnAGL9 and NnMADS6-like were determined to be located in the nucleus (Figure 5). 


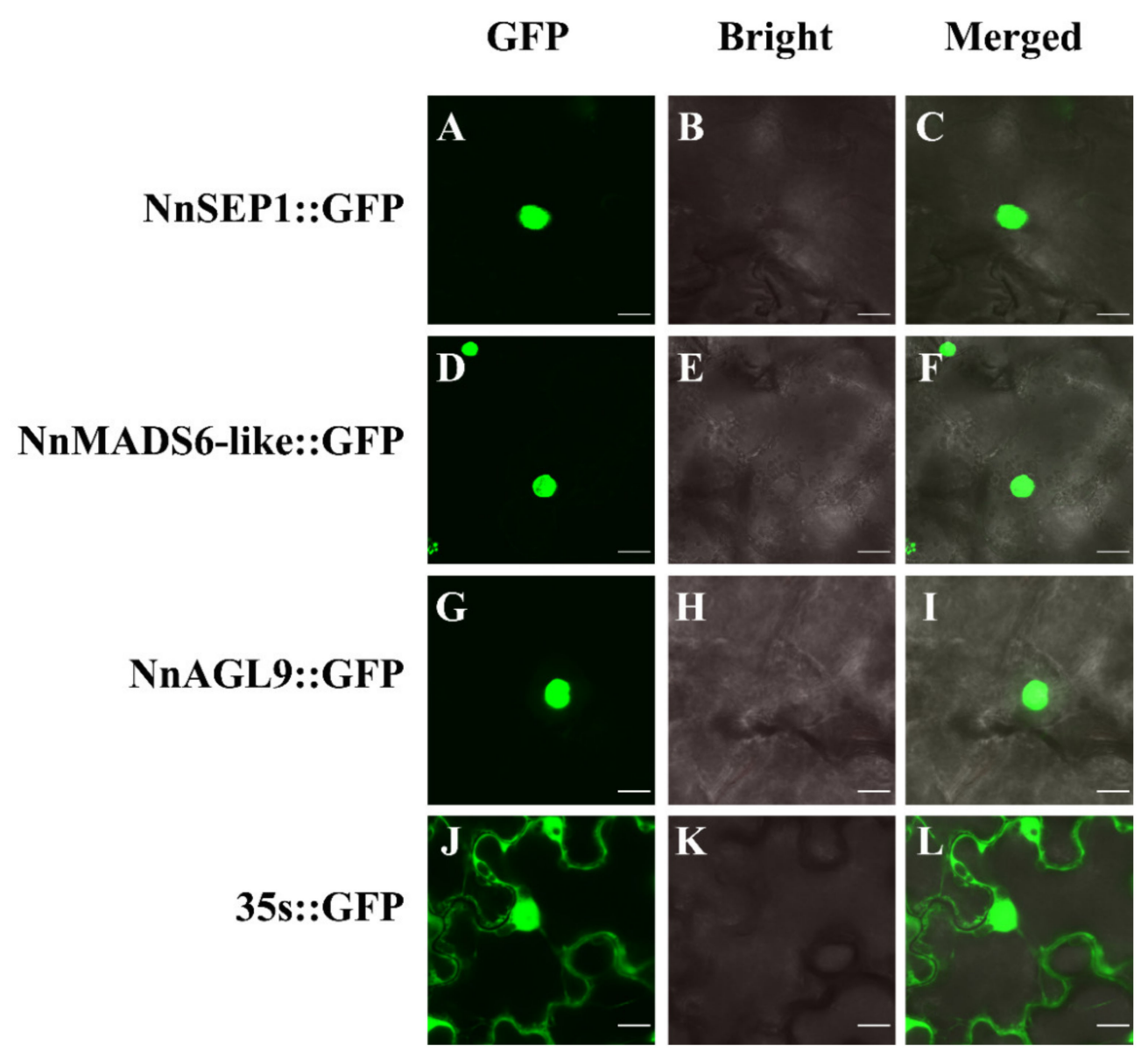

Figure 5. Subcellular localization of NnSEP1, NnMADS6-like and NnAGL9 in N. benthamiana. (A) 35S-NnSEP1-GFP was especially expressed in the nucleus of tobacco leaf cells. (B) The bright field of 35S-NnSEP1-GFP in tobacco leaf. (C) The merged field of GFP and the bright field of 35SNnSEP1-GFP. (D) 35S-NnMADS6-like-GFP was especially expressed in the nucleus. (E) The bright field of 35S- NnMADS6-like-GFP in tobacco leaf. (F) The merged field of GFP and the bright field of 35S- NnMADS6-like-GFP. (G) 35S-NnAGL9-GFP was especially expressed in the nucleus. (H) The bright field of 35S-NnAGL9-GFP in tobacco leaf. (I) The merged field of GFP and the bright field of 35S-NnAGL9-GFP. (J) 35S-GFP was especially expressed in the nucleus and plasma membranes. (K) The bright field of 35S-GFP in tobacco leaf. (L) The merged field of GFP and the bright field of 35S- GFP. Bar $=50 \mu \mathrm{m}$.

\subsection{Expression Pattern Analysis of NnSEP1, NnAGL9 and NnMADS6-like Genes}

In order to analyze the gene expression in the different plant tissues, we collected different parts of the single-flowered Asian lotus at the bud stage, including the root, pedicel, leaf, receptacle, rhizome and flower. All three genes were specifically expressed in the flower but hardly expressed in other organs (Figure S2). To further clarify the expression patterns of the three genes in floral tissues, we collected flower buds at four different growth stages (Figure S3) and divided them into sepals, petals, stamens and pistils for detection.

In the sepals, the expression patterns of the three genes were similar, but the expression of NnAGL9 was the lowest of the three genes (Figure 6A), which suggests that SEP1 and MADS6-like play an important role in sepal formation. In the petals, the expression level of NnMADS6-like and NnAGL9 reached the highest level at the mature stage. However, the expression of NnSEP1 did not change markedly in each period (Figure 6B). Expression patterns were similar for NnSEP1 and NnAGL9 in the stamens and pistils, while NnMADS6like was barely expressed in both tissues. In the stamens, the expression of NnMADS6-like 
was low, and the expression tendency of NnSEP1 and NnAGL9 was different in other tissues. The expression level of NnAGL9 reached the highest at stage II and the lowest at stage IV (Figure 6C). Although the expression of the genes in the pistils slightly fluctuated, their expression was not as high as in the stamens (Figure 6D).
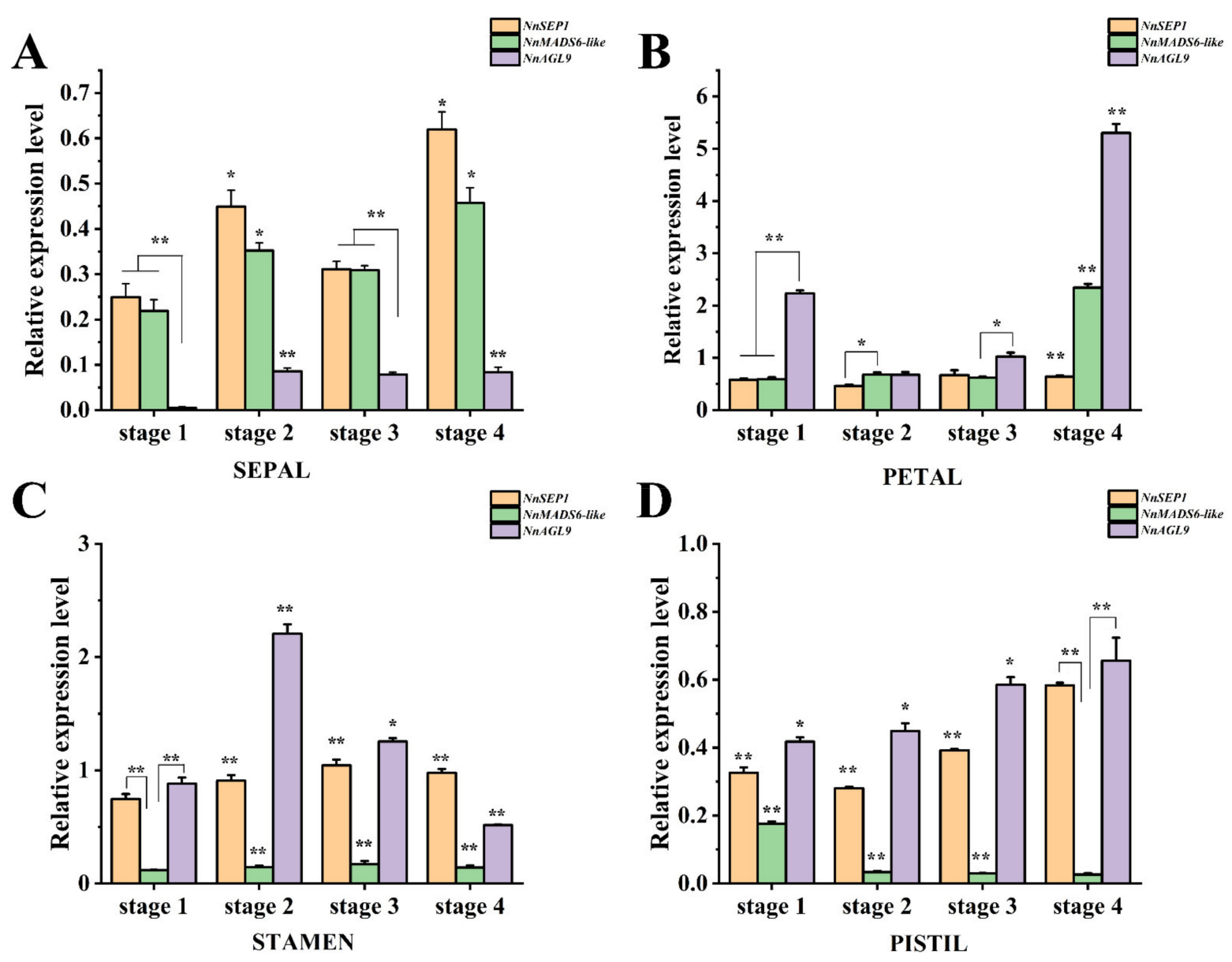

Figure 6. The expression pattern of three genes in different tissues at different floral development stages of N. nucifera. (A) Expression pattern of three genes in sepals. (B) Expression pattern of three genes in petals. (C) Expression pattern of three genes in stamens. (D) Expression pattern of three genes in pistils. The genes expression was detected using the RT-PCR method and NnACTIN as the internal reference gene. All data are means of three replicates, with error bars indicating standard deviations. The values are means $\pm \mathrm{SD}$ of triplicate analysis (* indicating $p<0.05,{ }^{* *}$ indicating $p<0.001$ ).

\subsection{Protein Interaction Between NnSEP1, NnMADS6-Like and NnAGL9}

Class $E$ genes play a fundamental role in floral organogenesis by binding different types of proteins. To identify the protein-protein interactions among NnSEP1, NnMADS6like and NnAGL9, an in vitro yeast two-hybrid (Y2H) assay was introduced to this study. The result shows NnMADS6-like interacted with NnAGL9 in yeast, no matter who was the bait. However, NnSEP1 can bind to NnMADS6-like and NnAGL9 when using NnSEP1 as the bait. On the contrary, NnSEP1 could not form a polymer when it was used as the prey (Figure 7). This infers that NnMADS6-like and NnAGL9 can form a complex interaction during the development of floral organs, while the function of NnSEP1 was different from NnMADS6-like and NnAGL9. However, more experimental evidence is needed to understand its biological function. 


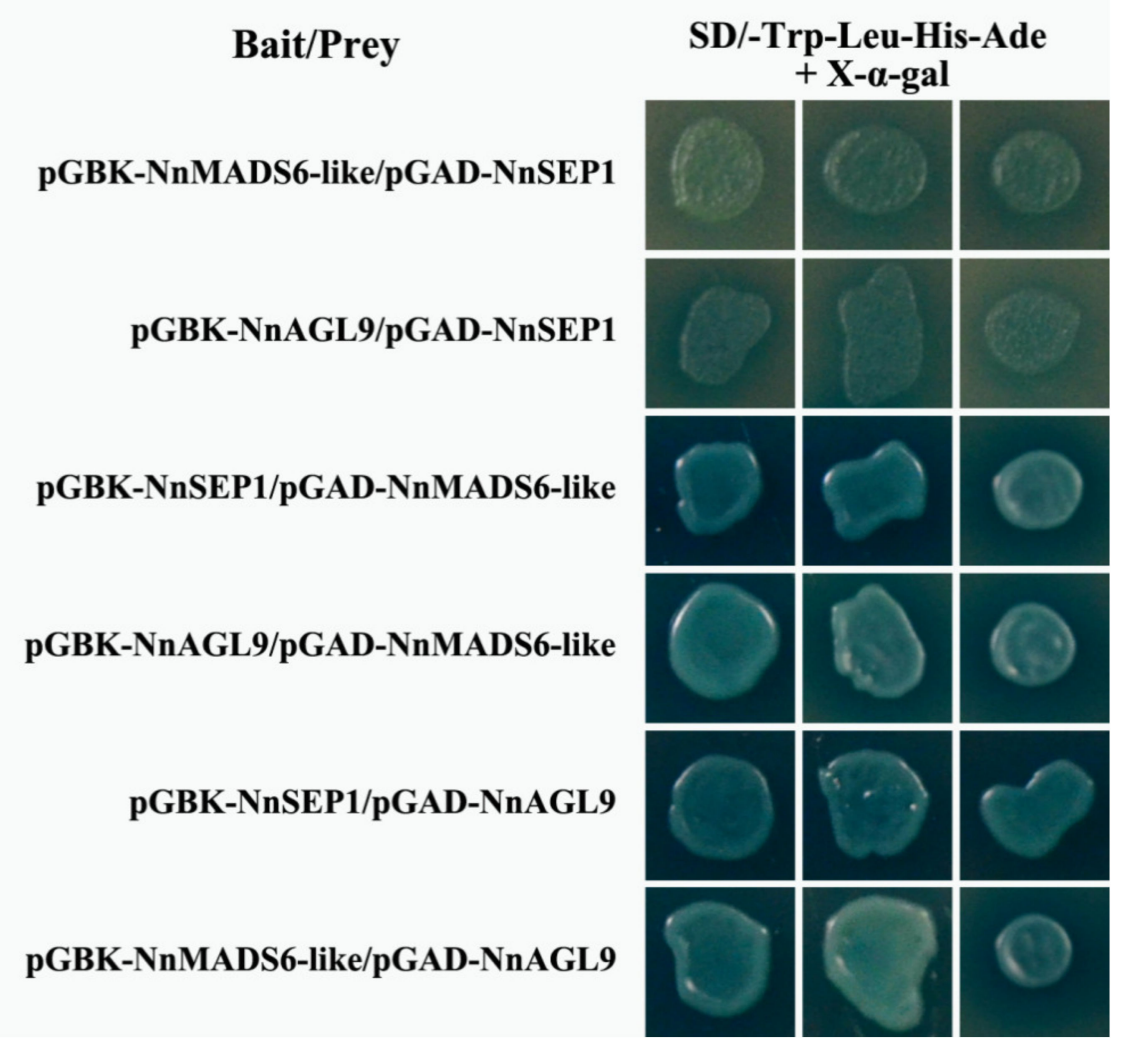

Figure 7. Protein interactions between NnSEP1, NnMADS6-like and NnAGL9 in vitro. Interactions were determined in yeast on selective medium SD/-Trp-Leu-His-Ade/X- $\alpha$-Gal, and the experiments were repeated three times with the same results. $\mathrm{AD}$ and $\mathrm{BK}$ clones containing empty vectors were used as the negative controls, shown in Figure S4.

\section{Discussion}

Floral organs are pivotal for flowering plants and controlled by intricate genetic networks. The MADS-box genes have been recruited as the primary elements of the genetic networks that control flower organ formation during plant evolution. Although several genes involved in lotus flower formation have been predicted, none of them, however, have been cloned and characterized with any function [33,34]. In this work, in order to understand the genetic relationship between the sepals and petals of the lotus, we cloned an $E$ class gene, NnSEP1. Unexpectedly, and fortunately, we also obtained two homologous genes NnAGL9 and NnMADS6-like from N. nucifera. Sequence homology analysis showed that NnSEP1, NnAGL9 and NnMADS6-like belonged to the MADS-box gene family. Their $5^{\prime}$-end sequences were very similar, but the $3^{\prime}$-end sequences were different. The expression pattern showed a significant difference in function between these two genes and SEP1. The localization prediction and subcellular localizations of NnSEP1, NnAGL9 and NnMADS6like showed that all three proteins were located in the nucleus. Previous studies showed that there were three SEP genes (SEP1, SEP2 and SEP3) in Arabidopsis which affected the development of floral organs [35-38]. When the three genes were mutated simultaneously, the floral organs were transformed into sepals. Additionally, the $B$ and $C$ genes need the SEP gene during pattern formation and organogenesis [19]. In determining the formation of lotus sepals, more experimental evidence is needed to confirm whether NnAGL9 and NnMADS6-like have a similar function to that of the NnSEP1 gene.

$A G L 6$ has a specific expression pattern in floral organs, but it varies slightly in different plant groups. Currently, the AGL6 homologous gene is considered as one of the four core participants in the plant regulatory network for flower development (SQUA-like, $D E F / G L O$-like, $A G$-like and AGL6/SEP1-like) [24]. It mainly has the functions of regulating the flowering time, determining the characteristics of the flower meristem, determining the 
characteristics of flower organs and in the development of flower organs. Phylogenetic tree analysis showed that AGL6 and SEP were sister branches [1,26]. The relationship between AGL6 and SEP was relatively close [38]. In many species such as Petunia $\times$ hybrida and Oryza sativa, the function of AGL6 was similar to the class $E$ gene family [26,39]. However, it has a different function in Oncidium flexuosum and Nymphaea tetragona. The function of AGL6 was similar to that of AP1 that belongs to the class $A$ gene family [40]. In this study, the pattern of the relatively high expression level of NnMADS6-like was similar to that of NnSEP1 in the sepals and petals, while the expression patterns in the stamens and pistils were different. This hints that NnMADS6-like might have a function as a class $A$ gene in N. nucifera.

The function of AGL9 is also known as SEP3 in the model plant Arabidopsis thaliana. Phylogenetic analysis illustrated that NnAGL9 has a close relationship with the SEP class. Smaczniak's research showed that the binding between SEP3 and other MADS-box proteins can change the chromatin state [16,41-43]. It was also suggested that SEP3, as a pioneer transcription factor, modifies chromatin accessibility [44]. Based on a $\mathrm{Y} 2 \mathrm{H}$ assay and gene expression patterns, a putative protein interaction showed that SEP3 interacted with AGL6 in the petals, stamens and pistils in Arabidopsis [45]. Our results also confirm that NnAGL9 interacts with NnMADS6-like in yeast, no matter who is the bait. It is suggested that NnAGL9 performs the function of SEP3 in the process of lotus organogenesis.

Although the lotus is a basal eudicot species in flowering plants, there are significant differences in the genes that control the sepals and petals. NnMADS6-like and NnSEP1 might determine the formation of lotus sepals. To further understand the function of NnSEP1, NnAGL9 and NnMADS6-like, we need to identify other factors and genes that are integrated in flowering development pathways and investigate how these regulators control floral organogenesis. Successfully deciphering the other class gene families and SEP genes will broaden our knowledge about the floral organ recognition control network in N. nucifera.

Supplementary Materials: The following are available online at https: / www.mdpi.com/article/ 10.3390/plants10081629/s1, Figure S1: The wild Asian lotus was introduced from Weishan Lake in Shandong Province, China, Figure S2: The gene expression in different tissues, Figure S3: The flower buds of Asian lotus at four different growth stages. The lengths of buds from left to right are $2 \mathrm{~cm}, 3.8 \mathrm{~cm}, 6.5 \mathrm{~cm}$ and $9.5 \mathrm{~cm}$; bar $=2 \mathrm{~cm}$, Figure S4: The negative controls of protein interaction in vivo. Interactions were determined in yeast on selective medium SD/-Trp-Leu-His-Ade, and the experiments were repeated in triplicates with the same results.

Author Contributions: Conceptualization, D.Z.; methodology, H.L. and W.Z.; software, Q.L. and H.L.; validation, W.Z., Q.L., H.L. and X.A.; formal analysis, original draft preparation, W.Z., X.A. and D.Z.; investigation, L.X. and Q.L.; resources, F.L.; data curation, Z.L.; writing-original draft preparation, H.L. and D.Z.; writing—review and editing, D.Z., D.T. and L.C.; funding acquisition, D.Z. and L.C. All authors have read and agreed to the published version of the manuscript.

Funding: This work was supported by funds from the National Natural Science Foundation of China (31572158, 31570300, 32072620); and the Shanghai Landscaping Administration Bureau (G202405, G192415)

Institutional Review Board Statement: Not applicable.

Informed Consent Statement: Not applicable.

Data Availability Statement: This study did not report any other data.

Acknowledgments: We thanks Wen Shao gave the support in Confocal microscopy \& image analysis.

Conflicts of Interest: The authors declare no conflict of interest with respect to authorship, research and publishing this article. 


\section{References}

1. Bowman, J.L.; Smyth, D.R.; Meyerowitz, E.M. The ABC model of flower development: Then and now. Development 2012, 139, 4095-4098. [CrossRef]

2. Norman, C.; Runswick, M.; Pollock, R.; Treisman, R. Isolation and properties of cDNA clones encoding SRF, a transcription factor that binds to the c-fos serum response element. Cell 1988, 55, 989-1003. [CrossRef]

3. Passmore, S.; Maine, G.T.; Elble, R.; Christ, C.; Tye, B.K. Saccharomyces cerevisiae protein involved in plasmid maintenance is necessary for mating of MAT alpha cells. J. Mol. Biol. 1988, 204, 593-606. [CrossRef]

4. Sommer, H.; Beltran, J.P.; Huijser, P.; Pape, H.; Lonnig, W.E.; Saedler, H.; Schwarz-Sommer, Z. Deficiens, a homeotic gene involved in the control of flower morphogenesis in Antirrhinum majus: The protein shows homology to transcription factors. EMBO J. 1990, 9, 605-613. [CrossRef] [PubMed]

5. Alvarez-Buylla, E.R.; Pelaz, S.; Liljegren, S.J.; Gold, S.E.; Burgeff, C.; Ditta, G.S.; Ribas-de Pouplana, L.; Martinez-Castilla, L.; Yanofsky, M.F. An ancestral MADS-box gene duplication occurred before the divergence of plants and animals. Proc. Natl. Acad. Sci. USA 2000, 97, 5328-5333. [CrossRef]

6. Parenicova, L.; de Folter, S.; Kieffer, M.; Horner, D.S.; Favalli, C.; Busscher, J.; Cook, H.E.; Ingram, R.M.; Kater, M.M.; Davies, B.; et al. Molecular and phylogenetic analyses of the complete MADS-box transcription factor family in Arabidopsis: New openings to the MADS world. Plant Cell 2003, 15, 1538-1551. [CrossRef]

7. Alvarez-Buylla, E.R.; Liljegren, S.J.; Pelaz, S.; Gold, S.E.; Burgeff, C.; Ditta, G.S.; Vergara-Silva, F.; Yanofsky, M.F. MADS-box gene evolution beyond flowers: Expression in pollen, endosperm, guard cells, roots and trichomes. Plant J. 2000, 24, 457-466. [CrossRef]

8. Zhang, H.; Forde, B.G. An Arabidopsis MADS box gene that controls nutrient-induced changes in root architecture. Science 1998, 279, 407-409. [CrossRef]

9. Huang, F.; Xu, G.L.; Chi, Y.J.; Liu, H.C.; Xue, Q.; Zhao, T.J.; Gai, J.Y.; Yu, D.Y. A soybean MADS-box protein modulates floral organ numbers, petal identity and sterility. BMC Plant Biol. 2014, 14. [CrossRef]

10. Pelucchi, N.; Fornara, F.; Favalli, C.; Masiero, S.; Lago, C.; Pe, M.E.; Colombo, L.; Kater, M.M. Comparative analysis of rice MADS-box genes expressed during flower development. Sex. Plant Reprod. 2002, 15, 113-122. [CrossRef]

11. Zhou, Y.; Hu, L.F.; Song, J.B.; Jiang, L.W.; Liu, S.Q. Isolation and characterization of a MADS-box gene in cucumber (Cucumis sativus L.) that affects flowering time and leaf morphology in transgenic Arabidopsis. Biotechnol. Biotechnol. Equip. 2019, 33 , 54-63. [CrossRef]

12. Vandenbussche, M.; Zethof, J.; Souer, E.; Koes, R.; Tornielli, G.B.; Pezzotti, M.; Ferrario, S.; Angenent, G.C.; Gerats, T. Toward the analysis of the petunia MADS box gene family by reverse and forward transposon insertion mutagenesis approaches: $\mathrm{B}, \mathrm{C}$, and $\mathrm{D}$ floral organ identity functions require SEPALLATA-like MADS box genes in Petunia. Plant Cell 2003, 15, 2680-2693. [CrossRef]

13. Seymour, G.B.; Ryder, C.D.; Cevik, V.; Hammond, J.P.; Popovich, A.; King, G.J.; Vrebalov, J.; Giovannoni, J.J.; Manning, K. A SEPALLATA gene is involved in the development and ripening of strawberry (Fragaria $\times$ ananassa Duch.) fruit, a non-climacteric tissue. J. Exp. Bot. 2011, 62, 1179-1188. [CrossRef]

14. Coen, E.S.; Meyerowitz, E.M. The war of the whorls: Genetic interactions controlling flower development. Nature 1991, 353, 31-37. [CrossRef]

15. Robles, P.; Pelaz, S. Flower and fruit development in Arabidopsis thaliana. Int. J. Dev. Biol. 2005, 49, 633-643. [CrossRef]

16. Smaczniak, C.; Immink, R.G.H.; Muino, J.M.; Blanvillain, R.; Busscher, M.; Busscher-Lange, J.; Dinh, Q.D.; Liu, S.J.; Westphal, A.H.; Boeren, S.; et al. Characterization of MADS-domain transcription factor complexes in Arabidopsis flower development. Proc. Natl. Acad. Sci. USA 2012, 109, 1560-1565. [CrossRef]

17. Theissen, G.; Saedler, H. Plant biology. Floral quartets. Nature 2001, 409, 469-471. [CrossRef]

18. Honma, T.; Goto, K. Complexes of MADS-box proteins are sufficient to convert leaves into floral organs. Nature 2001, 409, 525-529. [CrossRef]

19. Pelaz, S.; Ditta, G.S.; Baumann, E.; Wisman, E.; Yanofsky, M.F. B and C floral organ identity functions require SEPALLATA MADS-box genes. Nature 2000, 405, 200-203. [CrossRef]

20. Christensen, A.R.; Malcomber, S.T. Duplication and diversification of the LEAFY HULL STERILE1 and Oryza sativa MADS5 SEPALLATA lineages in graminoid Poales. Evodevo 2012, 3. [CrossRef]

21. Nishikawa, F.; Endo, T.; Shimada, T.; Fujii, H.; Shimizu, T.; Kobayashi, Y.; Araki, T.; Omura, M. Transcriptional changes in CiFT-introduced transgenic trifoliate orange (Poncirus trifoliata L. Raf.). Tree Physiol. 2010, 30, 431-439. [CrossRef]

22. Zhang, S.S.; Lu, S.J.; Yi, S.S.; Han, H.J.; Liu, L.; Zhang, J.Q.; Bao, M.Z.; Liu, G.F. Functional conservation and divergence of five SEPALLATA-like genes from a basal eudicot tree, Platanus acerifolia. Planta 2017, 245, 439-457. [CrossRef]

23. Shen, G.; Yang, C.H.; Shen, C.Y.; Huang, K.S. Origination and selection of ABCDE and AGL6 subfamily MADS-box genes in gymnosperms and angiosperms. Biol. Res. 2019, 52. [CrossRef] [PubMed]

24. Melzer, R.; Wang, Y.Q.; Theissen, G. The naked and the dead: The ABCs of gymnosperm reproduction and the origin of the angiosperm flower. Semin. Cell Dev. Biol. 2010, 21, 118-128. [CrossRef] [PubMed]

25. Morel, P.; Chambrier, P.; Boltz, V.; Chamot, S.; Rozier, F.; Bento, S.R.; Trehin, C.; Monniaux, M.; Zethof, J.; Vandenbussche, M. Divergent functional diversification patterns in the SEP/AGL6/AP1 MADS-Box transcription factor superclade. Plant Cell 2019, 31, 3033-3056. [CrossRef] [PubMed] 
26. Rijpkema, A.S.; Zethof, J.; Gerats, T.; Vandenbussche, M. The petunia AGL6 gene has a SEPALLATA-like function in floral patterning. Plant J. 2009, 60, 1-9. [CrossRef] [PubMed]

27. Chen, M.H.; Yan, T.X.; Shen, Q.; Lu, X.; Pan, Q.F.; Huang, Y.R.; Tang, Y.L.; Fu, X.Q.; Liu, M.; Jiang, W.M.; et al. Glandular trichome-specific wrky 1 promotes artemisinin biosynthesis in Artemisia annua. New Phytol. 2017, 214, 304-316. [CrossRef]

28. Zhang, Y.J.; Hao, X.Y.; Liang, Z.S.; Ke, W.D.; Guo, H.B. Efficient isolation of high-quality RNA from lotus Nelumbo nucifera ssp. nucifera tissues. Genet. Mol. Res. 2013, 12, 223-229. [CrossRef]

29. Xu, P.B.; Lian, H.L.; Xu, F.; Zhang, T.; Wang, S.; Wang, W.X.; Du, S.S.; Huang, J.R.; Yang, H.Q. Phytochrome B and AGB1 coordinately regulate photomorphogenesis by antagonistically modulating PIF3 stability in Arabidopsis. Mol. Plant. 2019, 12, 229-247. [CrossRef]

30. Saitou, N.; Nei, M. The neighbor-joining method-a new method for reconstructing phylogenetic trees. Mol. Biol. Evol. 1987, 4, 406-425. [CrossRef]

31. Tamura, K.; Nei, M. Estimation of the number of nucleotide substitutions in the control region of mitochondrial-dna in humans and chimpanzees. Mol. Biol. Evol. 1993, 10, 512-526. [PubMed]

32. Thompson, J.D.; Higgins, D.G.; Gibson, T.J. Clustal-W improving the sensitivity of progressive multiple sequence alignment through sequence weighting, position-specific gap penalties and weight matrix choice. Nucleic Acids Res. 1994, 22, 4673-4680. [CrossRef]

33. Lin, Z.Y.; Damaris, R.N.; Shi, T.; Li, J.J.; Yang, P.F. Transcriptomic analysis identifies the key genes involved in stamen petaloid in lotus (Nelumbo nucifera). BMC Genom. 2018, 19. [CrossRef]

34. Lin, Z.Y.; Liu, M.; Damaris, R.N.; Nyong'a, T.M.; Cao, D.D.; Ou, K.F.; Yang, P.F. Genome-Wide DNA methylation profiling in the lotus (Nelumbo nucifera) flower showing its contribution to the stamen petaloid. Plants 2019, 8, 135. [CrossRef]

35. Flanagan, C.A.; Ma, H. Spatially and temporally regulated expression of the MADS-box gene AGL2 in wild-type and mutant Arabidopsis flowers. Plant Mol. Biol. 1994, 26, 581-595. [CrossRef]

36. Ma, H.; Yanofsky, M.F.; Meyerowitz, E.M. AGL1-AGL6, an Arabidopsis gene family with similarity to floral homeotic and transcription factor genes. Genes Dev. 1991, 5, 484-495. [CrossRef]

37. Rounsley, S.D.; Ditta, G.S.; Yanofsky, M.F. Diverse roles for MADS box genes in Arabidopsis development. Plant Cell 1995, 7, 1259-1269. [CrossRef]

38. Endress, P.K. Evolutionary diversificatin of the flowers in angiosperms. Am. J. Bot. 2011, 98, 370-396. [CrossRef]

39. Ohmori, S.; Kimizu, M.; Sugita, M.; Miyao, A.; Hirochika, H.; Uchida, E.; Nagato, Y.; Yoshida, H. Mosaic floral organs1, an AGL6-like MADS box gene, regulates floral organ identity and meristem fate in rice. Plant Cell 2009, 21, 3008-3025. [CrossRef]

40. Zhang, L.S.; Chen, F.; Zhang, X.T.; Li, Z.; Zhao, Y.Y.; Lohaus, R.; Chang, X.J.; Dong, W.; Ho, S.Y.W.; Liu, X.; et al. The water lily genome and the early evolution of flowering plants. Nature 2020, 577, 79-84. [CrossRef]

41. Immink, R.G.H.; Tonaco, I.A.N.; de Folter, S.; Shchennikova, A.; van Dijk, A.D.J.; Busscher-Lange, J.; Borst, J.W.; Angenent, G.C. SEPALLATA3: The 'glue' for MADS box transcription factor complex formation. Genome Biol. 2009, 10. [CrossRef] [PubMed]

42. Jetha, K.; Theissen, G.; Melzer, R. Arabidopsis SEPALLATA proteins differ in cooperative DNA-binding during the formation of floral quartet-like complexes. Nucleic Acids Res. 2014, 42, 10927-10942. [CrossRef] [PubMed]

43. Melzer, R.; Theissen, G. Reconstitution of floral quartets in vitro involving class B and class E floral homeotic proteins. Nucleic Acids Res. 2009, 37, 2723-2736. [CrossRef]

44. Pajoro, A.; Madrigal, P.; Muino, J.M.; Tomas Matus, J.; Jin, J.; Mecchia, M.A.; Debernardi, J.M.; Palatnik, J.F.; Balazadeh, S.; Arif, M.; et al. Dynamics of chromatin accessibility and gene regulation by MADS-domain transcription factors in flower development. Genome Biol. 2014, 15. [CrossRef]

45. Malcomber, S.T.; Kellogg, E.A. SEPALLATA gene diversification: Brave new whorls. Trends Plant Sci. 2005, 10, 427-435. [CrossRef] 